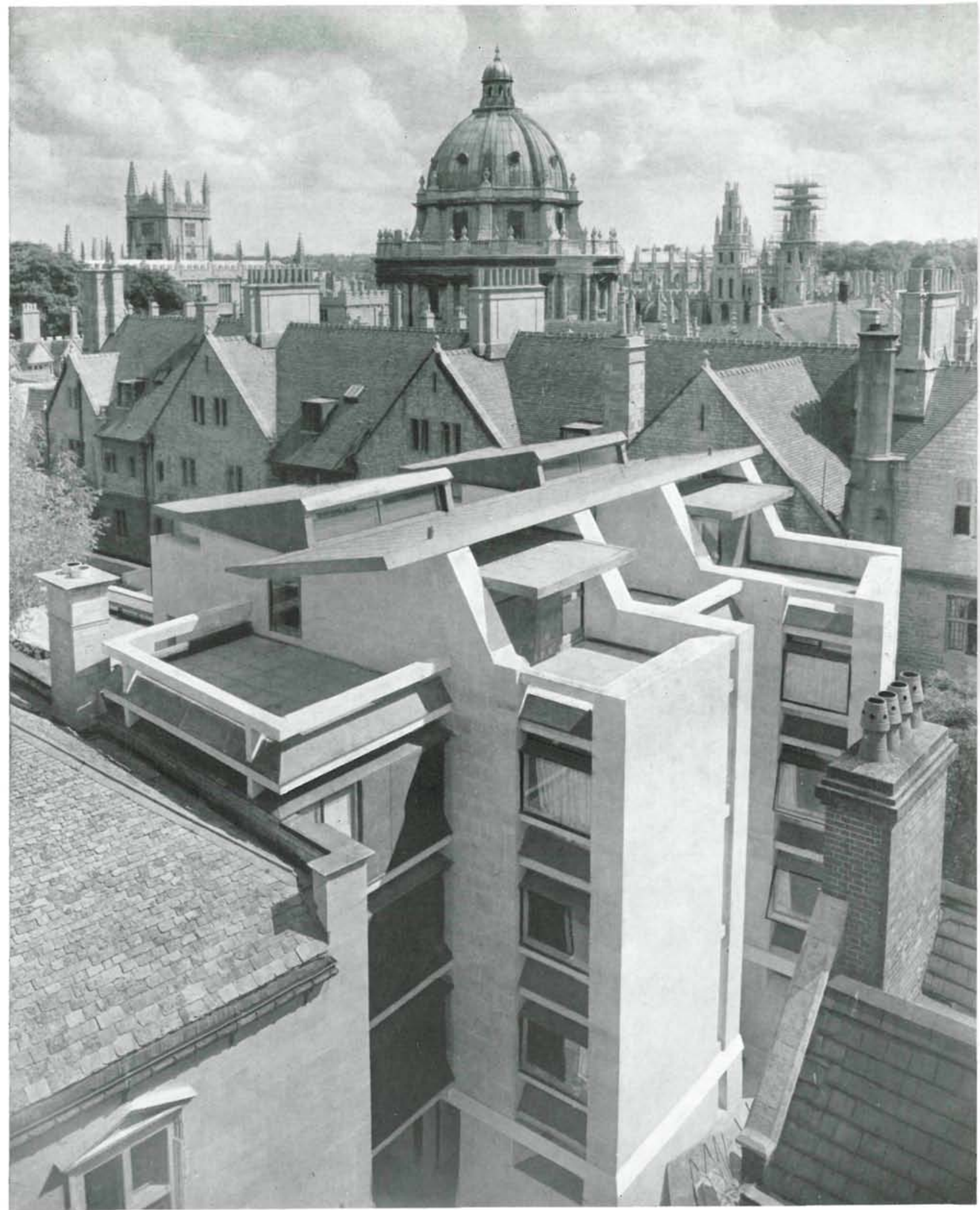

\title{
colegio brasemose
}

simepsis

POWELL Y MOYA

arquitectos

El lugar elegido para levantar los nuevos bloques fue el patio posterior del edificio, junto al Colegio Lincoln. Este terreno consiste en una zona ancha de $18,28 \times 18,28, y$ un pasaje de $33,52 \times 10,66 \mathrm{~m}$. Los edificios de los alreded
giano con una altura aproximada de 10,66 metros.

En la zona más ancha se levanta un edificio de cuatro plantas, con terraza, y en él 


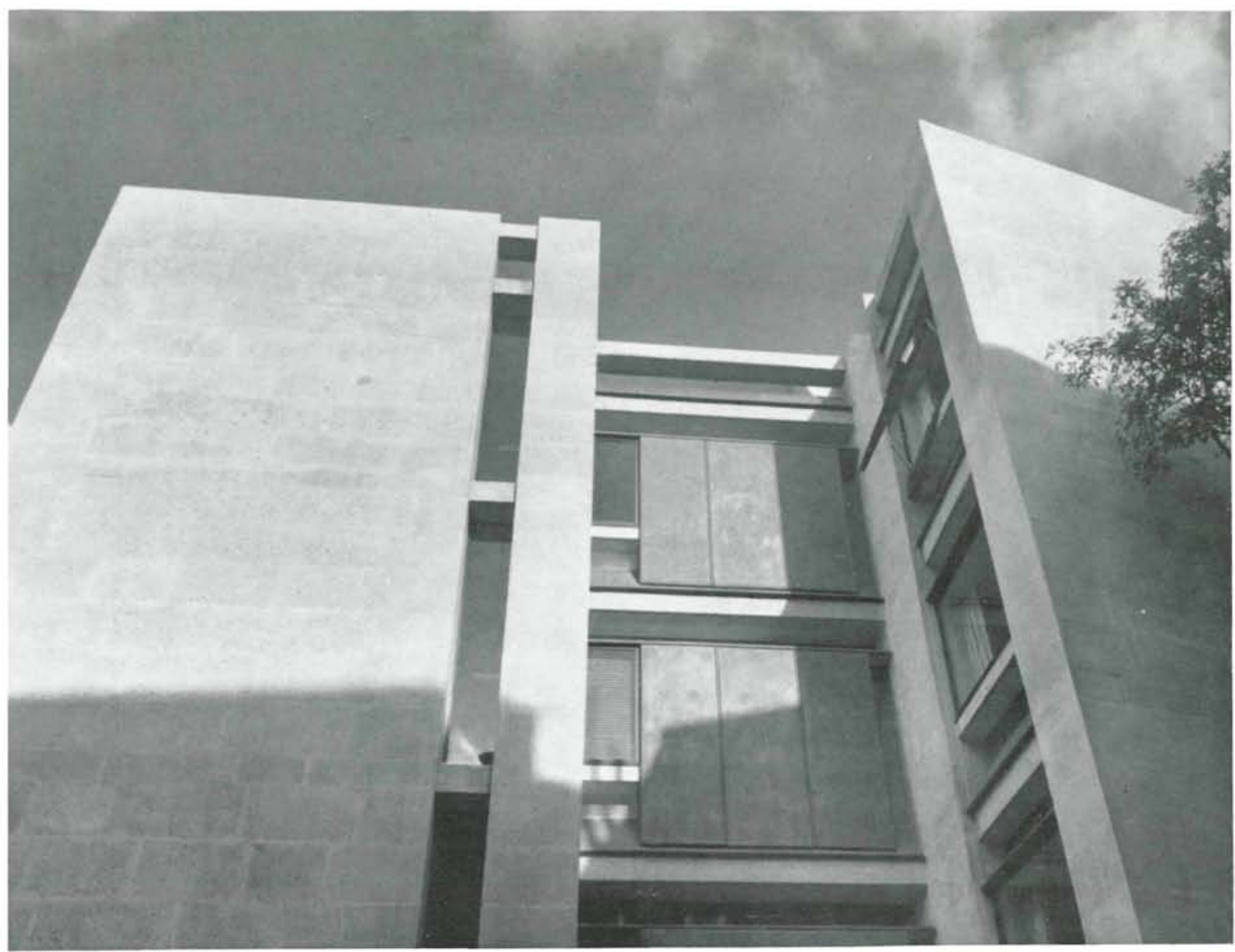

se han incluido 25 habitaciones. Los paisajes que se divisan y la silueta irregular de los edificios de los alrededores han influido notablemente en la elección de forma del bloque; se ha renunciado a un concepto rígidamente rectangular y se ha jugado con un perfil en diente de sierra, que ayuda a que las habitaciones sean tratadas individualmente, dotandolas de mayor encanto mediante la presentacion de paisajes oblicuos restringidos. $\mathbf{Y}$ asi, se han colocado ventanas en mas de un muro de cada habitación, de tal forma que, por una de ellas pueda gozarse del magnifico paisaje de tras que la otra permite el paso del sol.

En la zona estrecha no era posible construir edificios altos. Por lo tanto, se ha levantado un grupo de pabellones de una
sola planta, con uno o dos dormitorios en cada uno, hasta un total de siete. Las habitaciones tienen acceso por pequeños porches de entrada y recaen, por su parte posterior, hacia patinillos y jardines particulares.

E1 alto grado de perfeccionamiento conseguido en sus instalaciones contribuye a ampliar la comodidad de las habitacione y hacen de estos nuevos edificios una residencia cómoda agradable para los estudiantes que la ocupan.

El lugar elegido para levantar los nuevos bloques fue el patio posterior del edificio en su límite oeste, junto al Colegio Lincoln. Este terreno consiste en una zona ancha de $18,28 \times 18,28$ metros y un pasaje de, aproximadamente, $33,52 \times 10,66 \mathrm{~m}$. Los construidos en $1900 \mathrm{y}$ con una altura aproximada de 10,66 metros.

Más bien que dar a los arquitectos detallados programas de torios y cuartos de estar partiendo del reducido terreno que se les proporcionaba.

En la zona más ancha hay un edificio de cuatro plantas con una terraza, $\mathrm{y}$ en él se han incluido 25 habitaciones. Algunas de las mejores vistas son oblicuas. Seguramente los paisaje norte de uno de los jardines del Colegio Lincoln.

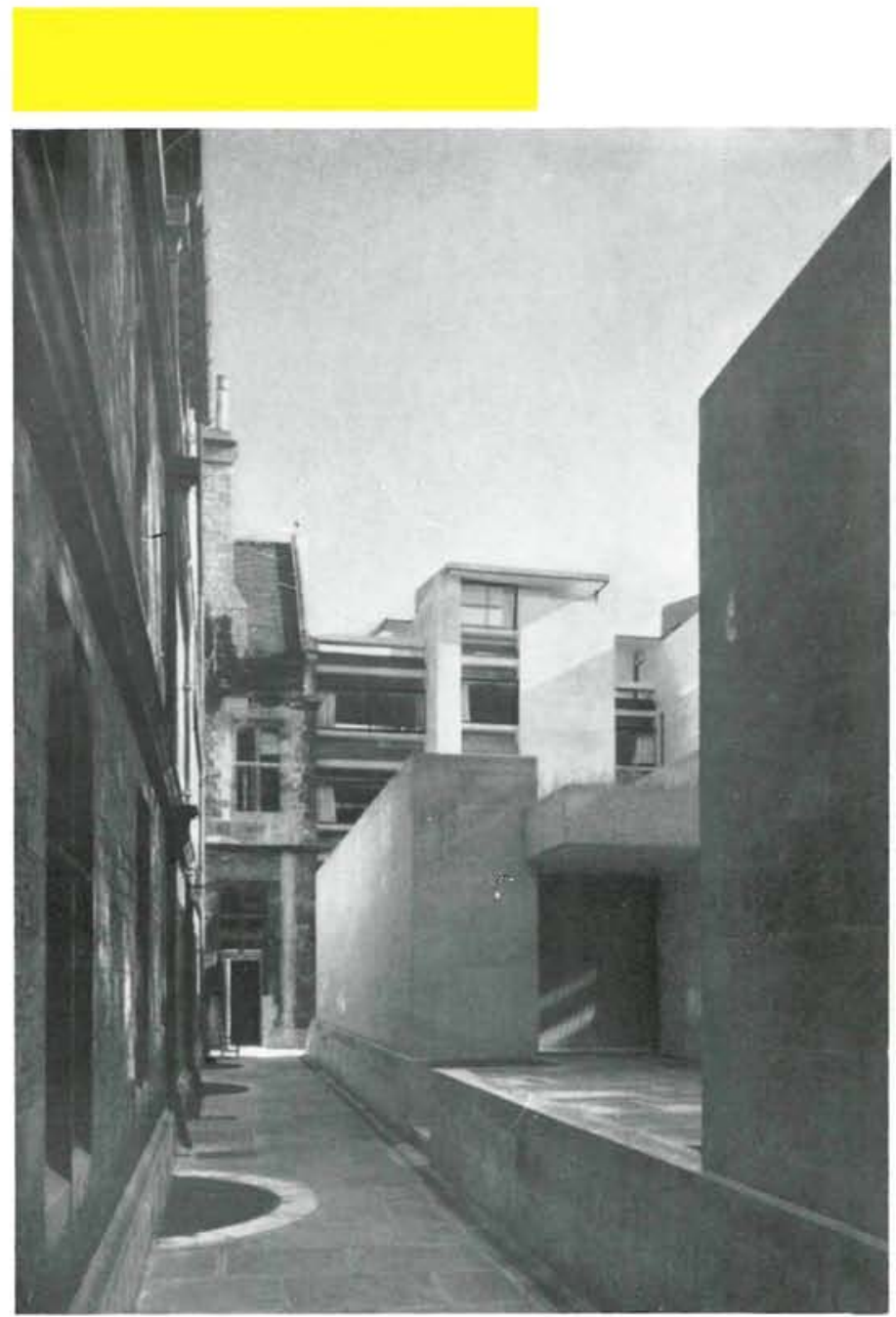



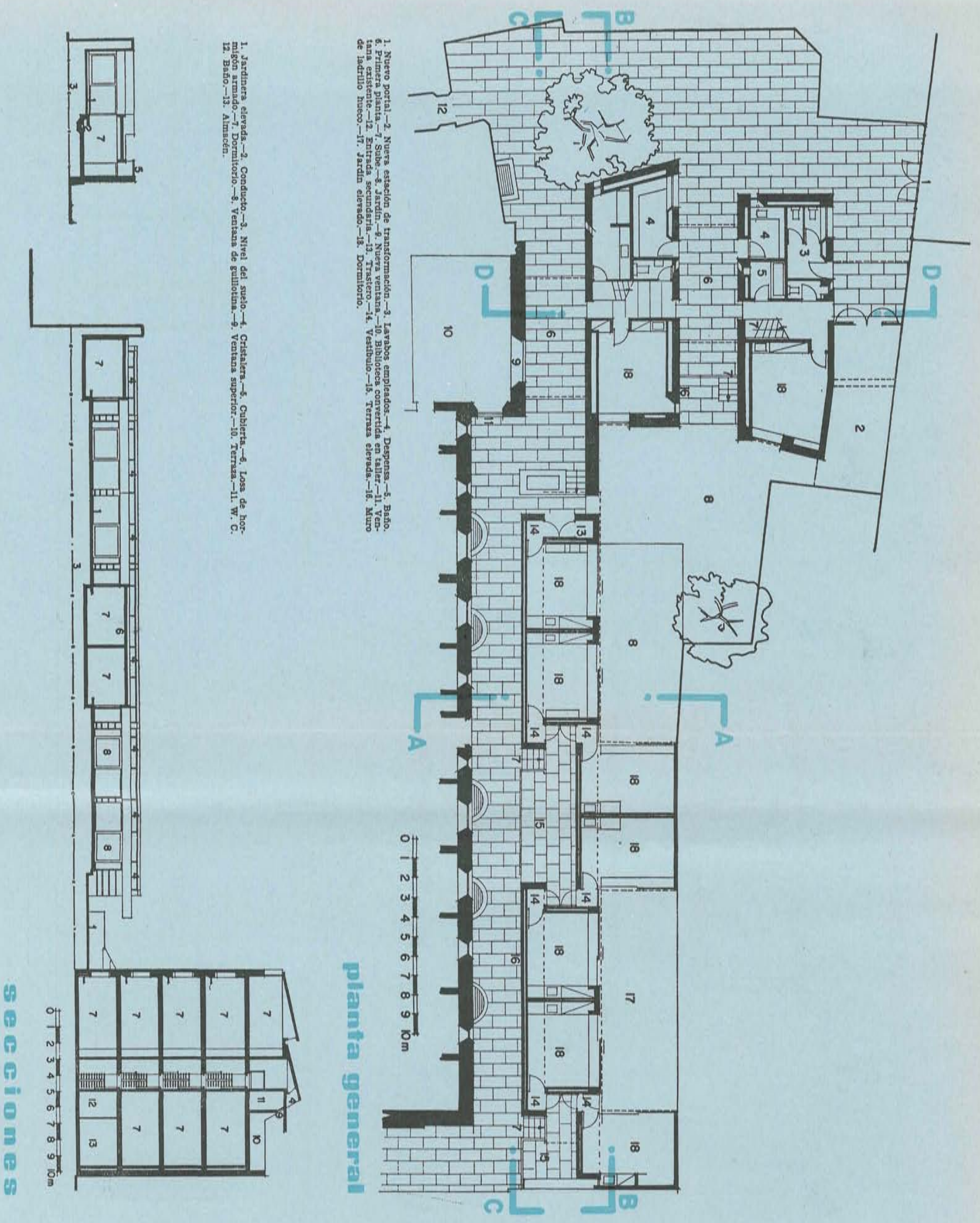

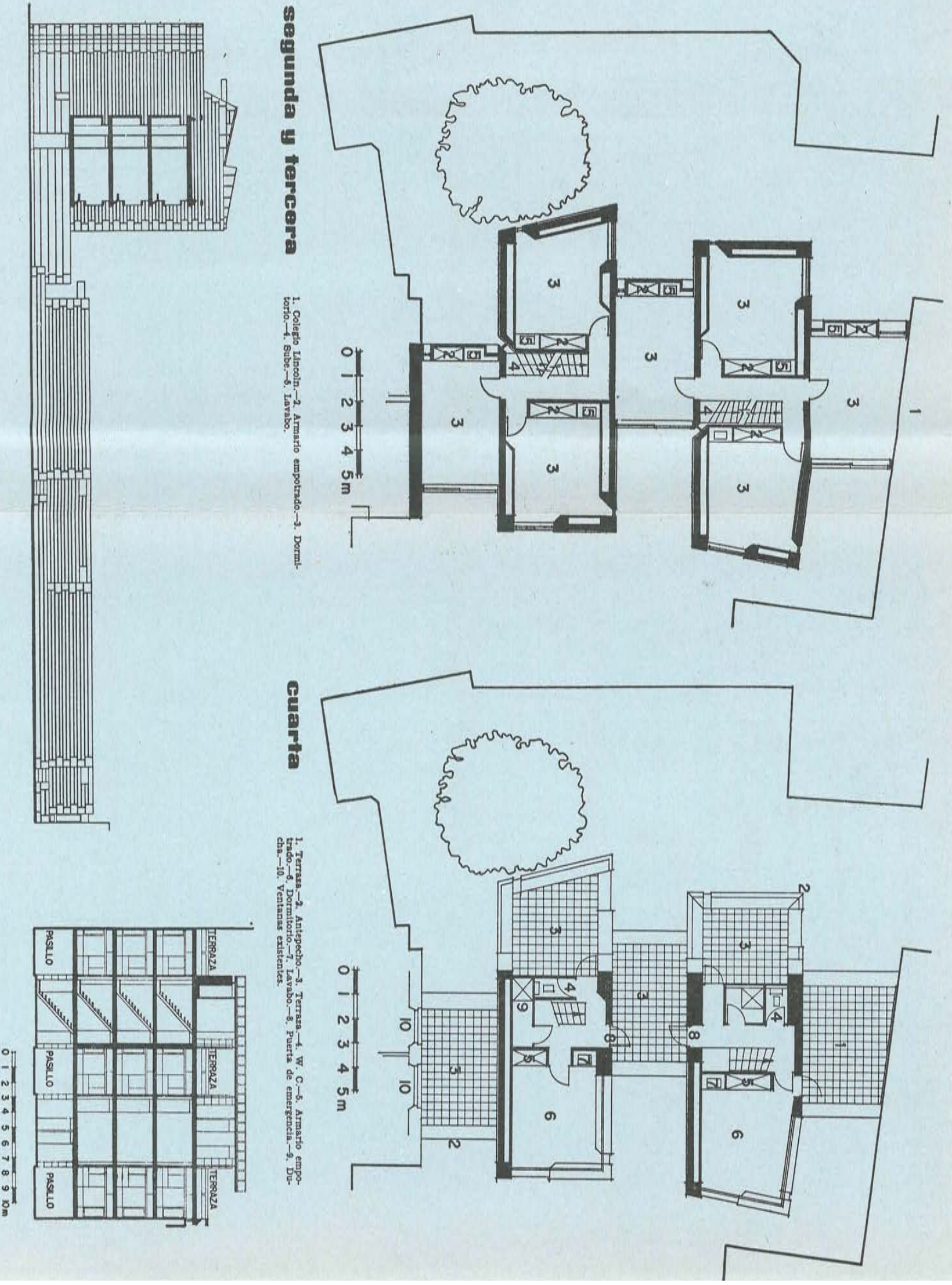


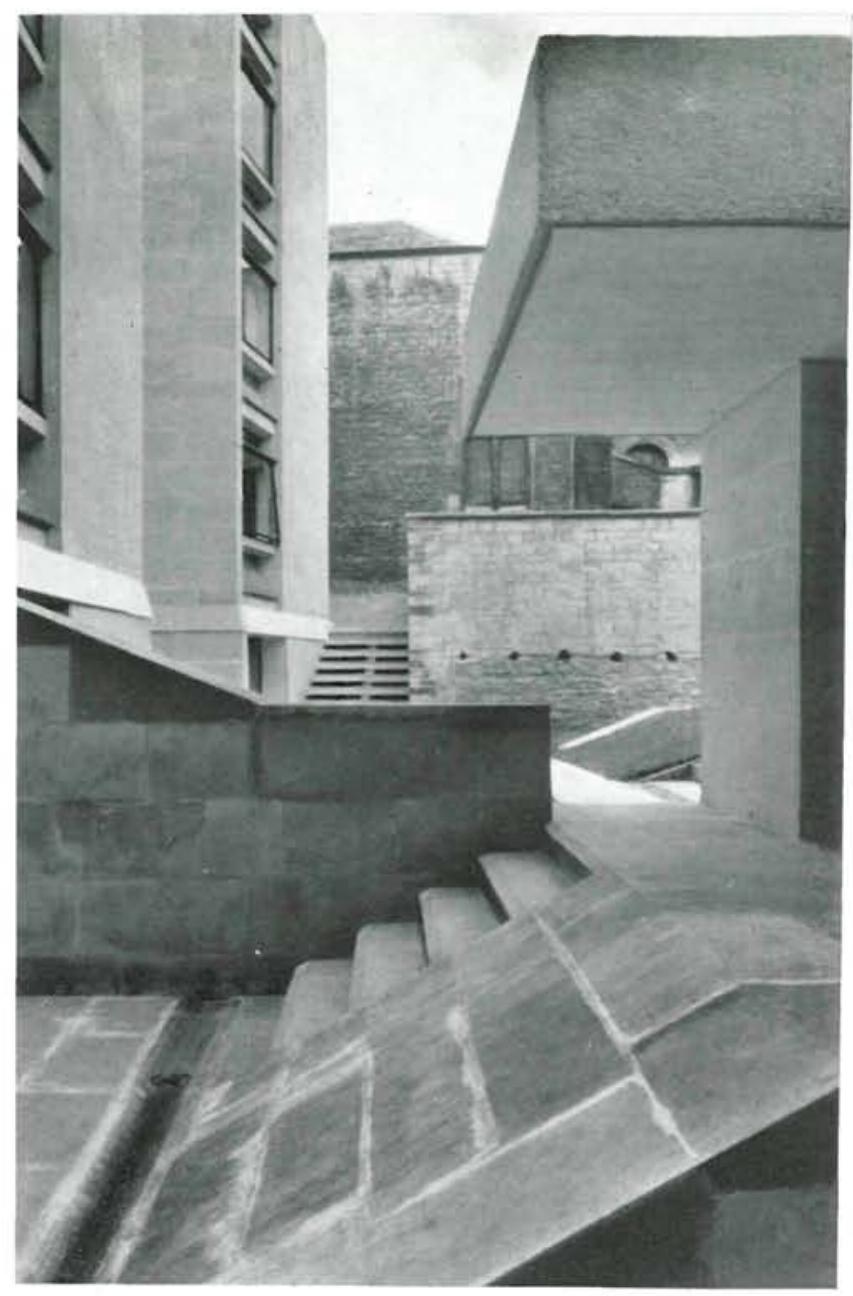

Estos paisajes y la silueta irregular de los edificios de los alrededores han influido notablemente en la elección de forma del bloque. Se ha renunciado a un plano rigidamente rectangular y se ha jugado con un perfil en diente de sierra, mas movido y mas en consecuencia con el caracter de los edificios vecinos y que ayuda a que las habitaciones sean tratadas individualmente con el fin de dotar a la mayoria de un encanto superior mediante la presentación de paisajes oblicuos y restringidos. Se han colocado ventanas en deas de un muro de cada nifico paisaje dal corma que por una de ellas pueda gozarse del magla luz solar de harte a ambos lados del pasillo para evitar la impresión de hotel,

En la zona estrecha no era posible construir edificios altos. Por lo tanto, se ha levantado un grupo de pabellones de una sola planta, conteniendo, cada uno, uno o dos dormitorios hasta un total de siete. Las habitaciones tienen acceso por pequeños porches de entrada, y recaen, por su parte posterior, hacia pequeños patios-jardines particulares.

En el proyecto, se ha atendido a respetar y armonizar la escala y materiales de los edificios de los alrededores. El exterior lleva piedra portland (que está siendo empleada actualmente en la mayoria de los trabajos de reconstruccion de edincios en Oxford, como material el peso del edificio. Esta piedra se ha utilizado con diferentes texturas, labras y tratamientos, según el lugar de colocación.

Estructura del edificio alto.-Los muros fundamentales se apoyan sobre durmientes soportados por pilares de hormigón armado, que, a su vez descansan sobre soleras de hormigón armado con las bases de hormigón en masa.

Los muros exteriores de carga están compuestos por ladrillos de $0,22 \mathrm{~m}$ de espesor y piedra portland de $0,05 \mathrm{~m}$. Los tabiques interiores no tienen carácter resistente y están formados por bloques de clínker
con mortero.

Los forjados son de hormigón armado «in situn con mantas de fibra de vidrio como aislamiento enrasados con cemento y, generalmente, ol mis con piezas de lante filte especiales. La parte inferior de los voladizos es de madera.

Las piezas inclinadas en las ventanas son de hormigón prefabricado, con arido de cuarcita blanca de Noruega, ligeramente abujardadas. Los paneles de las ventanas han sido tratados con albayalde y óxido de plomo para hacerlos opacos. Los cercos de las ventanas son de aluminio anodizado. Por último, los tramos de las escaleras son de madera dura.
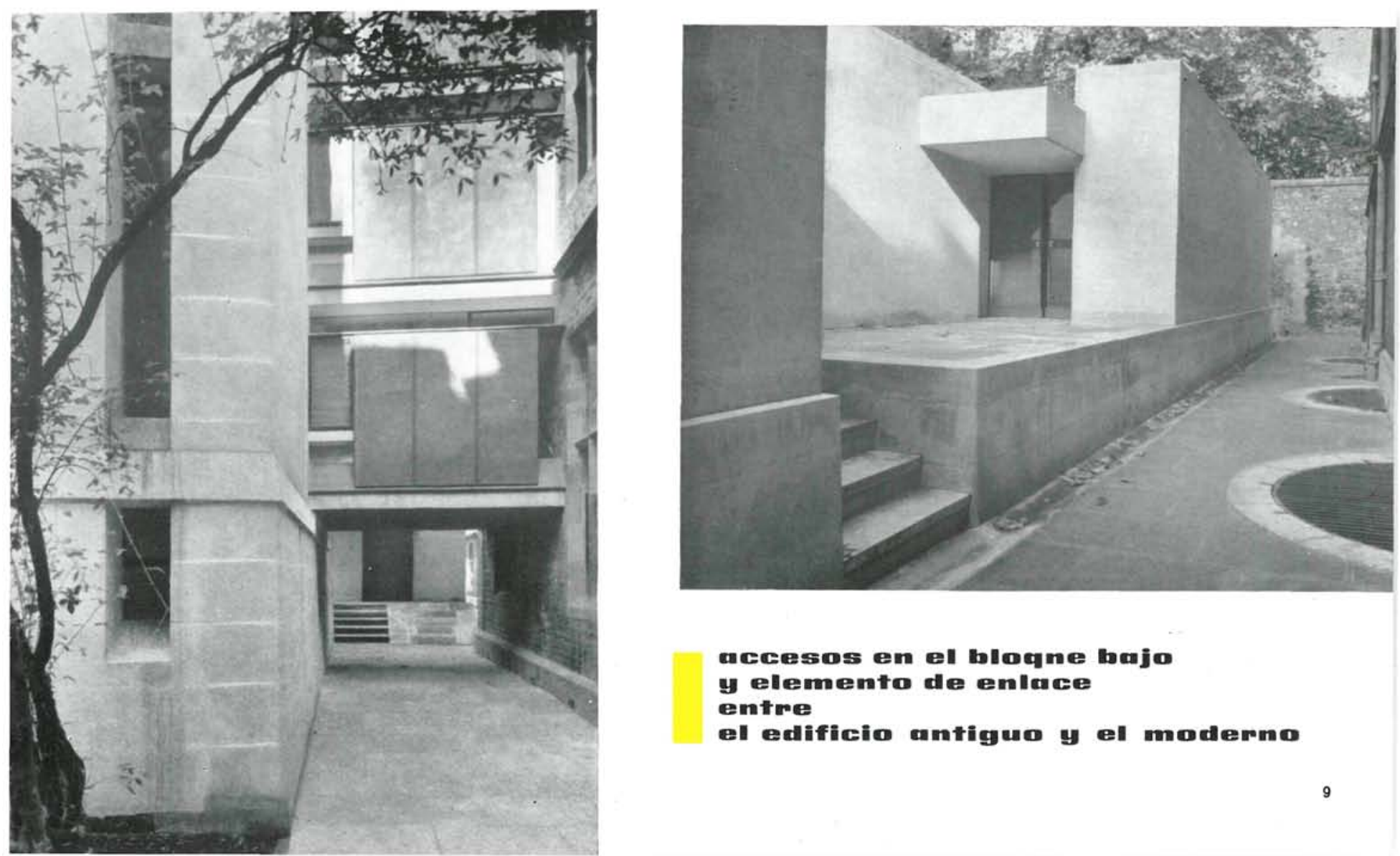

\author{
cocesos en el blocme bujo \\ y elemento die enlace \\ entre \\ El Edificio camtiguo y el moderno
}




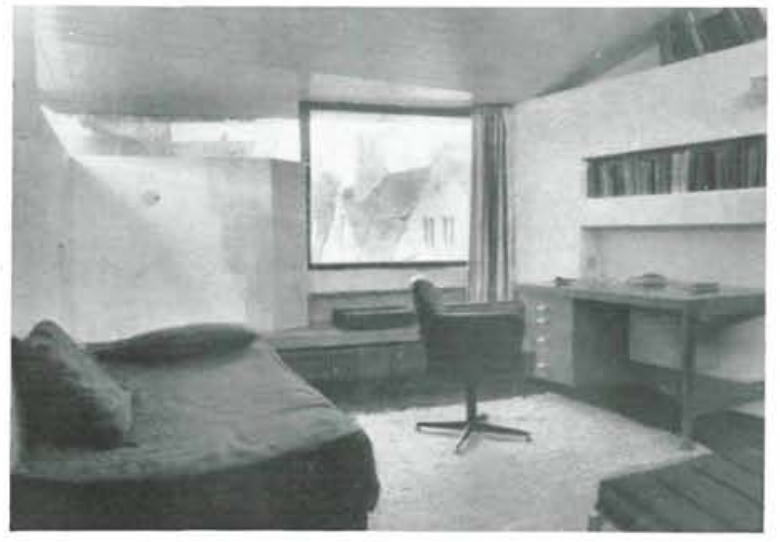

Estructura del edificio bajo,-La construcción es, en general, similar a la del edificio mas alto, excepto en los cimientos, que, en este caso, consisten en placas de hormigon armado sobre los muros primitivos, descansando sobre pilares y bóvedas.

Los muros de carga exteriores son huecos, con la superficie vista acabada de la misma manera que los del edificio más alto.

El sistema de calefacción es el mismo en ambas construcciones: central, con alimentación desde una sala de calderas existente anteriornas y suplementada con paneles radiantes eléctricos.

Todas estas instalaciones contribuyen a ampliar la comodidad de un residencia cómoda y agradable para los estudiantes que la ocupan.

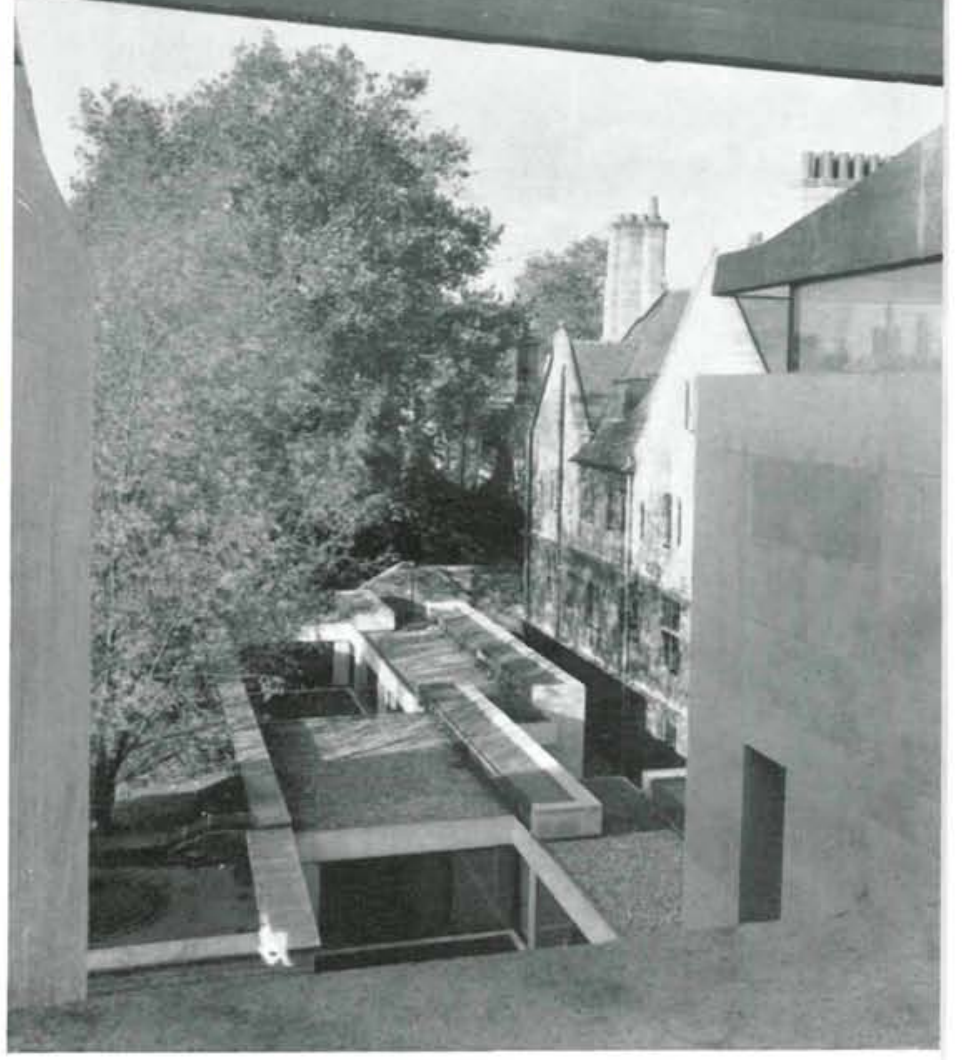

Fotor ERIC DE MARA BURGH GAIWEY Y J, V. L. HOPKINS

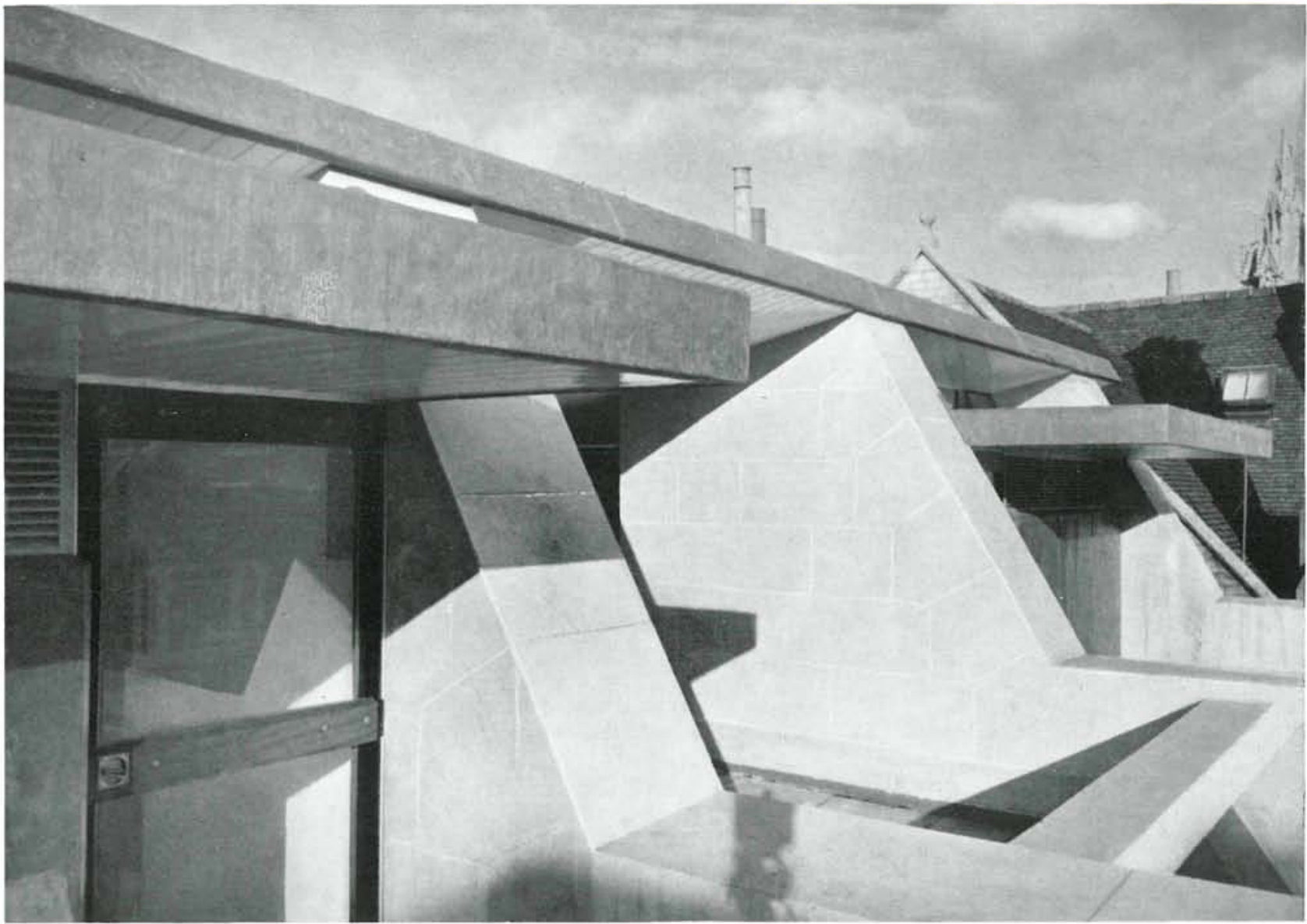




\section{Bollége Bursemose, Dxformat}

\section{NOUVEAUX EDIFICES}

Powell et Moya, architectes.

L'endroit choisi pour construire ces nouveaux bâtiments a été la cour postérieure de l'édifice, à côté du collège Lincoln. Ce terrain est une ample zone de $18,28 \times 18,28 \mathrm{~m}$ et un passage de $33,52 \times 10,66 \mathrm{~m}$. Les édifices des alentours sont de style gothique et géorgien d'une hauteur approximative de 10,66 m.

Dans la zone la plus large s'élève un édifice de quatre étages munis d'une terrasse, qui comprend 25 chambres. Le paysage et la silhouette irrégulière des édifices des alentours ont influé notablement sur le choix de la forme de l'édifice. On a renoncé à un concept rigidement rectangulaire pour jouer avec un profil en dent de scie qui a permis que les chambres soient traitées individuellement, en les dotant ainsi d'un charme particulier grâce à la vue de petits paysages obliques. L'une des fenêtres jouit du magnifique paysage en direction du Nord, alors que l'autre laisse entrer le soleil.

Il n'a pas été possible de construire d'édifices élevés, dans la zone étroite. C'est pourquoi on a eu recours à un groupe de pavillons d'un seul étage, d'une ou deux chambres chacun. On accède à ces chambres par de petits porches d'entrée et leur partie postérieure donne sur des courettes et des jardins particuliers.

Le perfectionnement, auquel on est arrivé pour les installations de ces bâtiments, les a dotés d'une commodité incomparable et fait de ces nouveaux édifices une résidence confortable et agréable.

\section{Brensemose Eollege, Oxford}

\section{NEW BUILDINGS}

Powell and Moya, architects.

The site chosen for the new blocks was the space behind the existing building, close to Lincoln College. This ground consists of a wide plot, of $18.28 \mathrm{~ms}$ by $18.28 \mathrm{~ms}$, plus a rectangle of $33.52 \mathrm{~ms}$ by $10.66 \mathrm{~ms}$. The surrounding buildings are in Gothic and Georgian style, and have a mean height of $11 \mathrm{~ms}$.

In the wider zone a building of four storeys has been constructed, with a terrace, and it includes 25 rooms. The surrounding view and the irregular outline of the neighbouring buildings has notably influenced the choice of shape for this block. A rigid rectangular shape has been avoided, and a kind of serrated outline has been achieved, by treating each room individually, and providing each of them with oblique and limited views of the surrounding buildings. To do this windows have been fitted to more than one wall of each room: one of them looks out over the magnificent north scenery, whilst the other lets in the sun.

In the narrow zone it became impossible to accommodate a tall building. Hence a number of one storey pavillions have been erected, housing one or two bedrooms each, seven in all. Access to the rooms is along small entrance porches, whilst at the back they open onto small private gardens.

The high standard of attention to detail in all the installations and services contributes to the comfort of the rooms and makes this into comfortable and pleasant living quarters for the students who occupy them.

\section{Schulle Brosemose, Oxfored}

\section{NEUE GEBÄUdE}

Powell und Moya, Architekten.

Der auserwählte Ort zur Erhebung der neuen Blöcke war der frühere Hof des Gebäudes, neben der Schule Lincoln. Dieses Gelände besteht aus einem weiten Teile von $18,28 \times 18,28$ und einem Durchgang von $33,52 \times 10,66$ $\mathrm{m}$. Die Gebäude der Umgebung sind im gotischen und georginischen Stile mit einer annähernden Höhe von $10,66 \mathrm{~m}$.

In der breitesten Zone erhebt sich ein Gebäude von 4 Stockwerken mit Terrasse, in dem sich 25 Zimmer befinden. Die Landschaften, die man undeutlich wahrnimmt, und der unregelmässige Schattenriss der Häuser ringsherum haben bemerkenswert die Auswahl der Blockform beeinflusst. Man hat auf einen streng rechtwinkligen Begriff verzichtet und mit einem Sheddach - Profil gespielt, was dazu beitrug, dass die Zimmer einzeln behandelt wurden, indem sie mit grösserem Reiz versehen wurden mittels der Darstellung von schiefen und eingeschränkten Landschaften. So wurden Fenster an mehr als einer Wand eines jeden Zimmers angebracht, derart, dass man durch eines derselben die herrliche Landschaft im Norden geniessen kann, während das andere den Eintritt der Sonne erlaubt.

In dem schmalen Teil war es nicht möglich, hohe Gebäude zu bauen. Deshalb wurden eine Gruppe von einstöckigen Gartenhäusern errichtet, mit einem oder zwei Schlafräumen in jedem, sieben im Ganzen. Die Zimmer haben einen Zugang durch eine kleine Vorhalle und fallen mit ihrer Rückseite auf kleine Höfe und Privatgärten ab.

Der hohe an den Anlagen erreichte Grad der Vollkommenheit trägt zu einer grösseren Bequemlichkeit der Zimmer bei und macht aus diesen neuen Gebäuden ein bequemes und angenehmes Wohnheim für die Stu. denten, die es bewohnen. 